\title{
Kompakt, rostfrei und wartungsfreundlich
}

\section{Ein neues Modell von Doppelmembranpumpen erlaubt einen wartungsarmen Betrieb mit minimaler Pulsation für einen gleichmäßigen Materialstrom beim Fördern und Spritzen.}

Eine neu entwickelte Membranpumpe mit einem Druckübersetzungsverhältnis von $3: 1$ ermöglicht eine günstige Alternative zu etablierten Pumpensystemen. Das neue Pumpenmodell Twin315 von Dete Dr. Tettenborn verfügt über PTFE-Verbundmembranen. Alle medienberührten Teile sind aus Edelstahl gefertigt. Somit ist die Pumpe sowohl für Lösemittel- als auch Wasserlacke, aber auch für andere Medien sehr gut geeignet. Durch das Druckübersetzungsverhältnis von 3:1 können Materialdrücke bis 21 bar bei einer Förderleistung von bis zu $3 \mathrm{l} / \mathrm{min}$ erreicht werden.

Darüber hinaus bietet das Modell die Vorteile einer druckübersetzten Membranpumpe, wie zum Beispiel eine gute Spülbarkeit und kompakte Bauweise, insbesondere aber deren geringe Pulsation. Deshalb eignet sich die Pumpe optimal für Ringleitungen und Farbversorgungssysteme.

Wie alle Pumpen von Dete entspricht auch die neue Membranpumpe der ATEX-Richtlinie 2014/34/EU und kann in Ex-Bereichen der Zone 1 eingesetzt werden. Typische Anwendungsgebiete der Pumpenmodelle liegen bei Wasser- beziehungsweise Lösemittellacken, scherempfindlichen Materialien und niedrig- bis hochviskosen Fluiden. Zu- dem ist der Transport über weite Förderstrecken möglich.

Das selbstansaugende System gewährleistet eine vereinfachte Inbetriebnahme. Das Gerät ist auf einen wartungsarmen Betrieb ausgelegt, eine Schmierung ist nicht erforderlich. Diese Vorteile zusammen mit einer Vielzahl an Zubehör, wie verschiedenen Ansaugsystemen und Montagearten, machen die Doppelmembranpumpe zu einem universell einsetzbaren System.

Das Modell erlaubt eine Wand- oder Deckelmontage und steht in fahrbaren Versionen auf Pumpenkarren mit verschiedenen Ansaugsystemen - starr, flexibel oder mit Oberbehälter - zur Verfügung. Die Pumpe wird in Deutschland gebaut, alle Ersatzteile sind bei Dete am Standort Nürnberg bevorratet, sodass eine schnelle Reaktion im Reparaturfall gesichert ist. //

\section{Kontakt}

\section{Dete Dr. Tettenborn GmbH}

Nürnberg

Tel. 091134770

dete@dete.de,www.dete.de

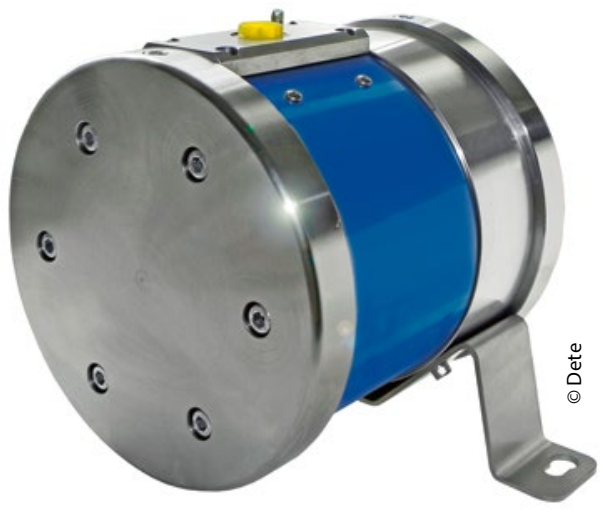

Die druckübersetzte Membranpumpe kann Materialien mit bis zu 2,1 MPa fördern. Ihre Förderkammern bestehen aus Edelstahl, bestückt mit PTFE-Membranen. 